\title{
MAXIMAL TRIADS AND PRIME DECOMPOSITIONS OF SURFACES EMBEDDED IN 3-MANIFOLDS
}

\author{
MICHAEL MOTTO
}

\begin{abstract}
In 1975, Suzuki proved that prime decompositions of connected surfaces in $S^{3}$ are unique up to stable equivalence of the factors. This paper extends his result to a large class of 3-manifolds, and demonstrates that this result does not apply to all 3-manifolds. It also answers a question he raised by showing that it is possible for inequivalent surfaces in $S^{3}$ of the same genus to be stably equivalent. The techniques used involve the notion of Heegaard splittings of 3-manifold triads.
\end{abstract}

In 1975, Suzuki proved in [Su] that prime decompositions of connected surfaces in $S^{3}$ are unique up to stable equivalence of the factors. This paper will extend his result to all two-sided surfaces (not necessarily connected) embedded in 3-manifolds which do not possess any nonseparating 2-spheres. It will also provide a counterexample demonstrating that this result does not apply to 3-manifolds which do possess nonseparating 2-spheres.

The goals of the first two sections are to summarize material that appears elsewhere, and to establish the conventions to be used here. The third section describes the maximal triad for a surface embedded in a 3-manifold, which is the primary tool that will be used in our proofs. Section 4 is devoted to proving the main results. The last section takes a closer look at stable equivalence of surfaces in $S^{3}$, and answers a question raised by Suzuki in [Su].

\section{SURfaces, CONNECTED SUMS, AND STABILIZATION}

Throughout this paper, the term surface will refer to a pair $(M, F)$, where $M$ is a compact, connected 3-manifold, and $F$ is a compact 2-manifold which is tame, two-sided, and properly embedded in $M$. Certain special surfaces are the following. $\left(S^{3}, S^{2}\right)$ is a 2 -sphere embedded in $S^{3} .\left(S^{3}, T^{2}\right)$ will denote an unknotted torus in $S^{3}$, one which bounds a solid torus on both sides, or in other words, a torus which forms a Heegaard splitting of $S^{3} .\left(B^{3}, D^{2}\right)$ will denote a disk properly embedded in a 3-ball.

We define a binary operation \# for surfaces, called connected sum. Let $\left(M_{1}, F_{1}\right)$ and $\left(M_{2}, F_{2}\right)$ be two surfaces. For $i=1,2$, let $B_{i} \subset$ Int $M_{i}$ be a tame 3-ball such that $\left(B_{i}, B_{i} \cap F_{i}\right) \cong\left(B^{3}, D^{2}\right)$. Let

$$
h:\left(\partial B_{1}, F_{1} \cap \partial B_{1}\right) \rightarrow\left(\partial B_{2}, F_{2} \cap \partial B_{2}\right)
$$

Received by the editors March 28, 1990.

1980 Mathematics Subject Classification (1985 Revision). Primary 57N10.

Key words and phrases. Connected sum, Heegaard splitting, maximal triad, prime decomposition, stable equivalence, surface. 
be a homeomorphism. Construct a surface $(M, F)$ as follows. Let $M$ be the manifold obtained by gluing $M_{1}-\operatorname{Int} B_{1}$ and $M_{2}-\operatorname{Int} B_{2}$ using $h$, and let $F$ be the manifold obtained by gluing $F_{1}-\operatorname{Int} B_{1}$ and $F_{2}-\operatorname{Int} B_{2}$ using $h$ restricted to $F_{1} \cap \partial B_{1}$. We write $(M, F)=\left(M_{1}, F_{1}\right) \#\left(M_{2}, F_{2}\right)$, and refer to $\left(M_{1}, F_{1}\right)$ and $\left(M_{2}, F_{2}\right)$ as factors of the connected sum. This operation is not well-defined due to the arbitrary nature of the component of $F_{i}$ which $B_{i}$ intersects, and since there are four choices (up to isotopy) of homeomorphism $h$ for any choice of $B_{1}$ and $B_{2}$. Of course, in some cases different choices of $B_{1}, B_{2}$, and $h$ will yield surfaces that are homeomorphic. For example, the connected sum of $n$ copies of $\left(S^{3}, T^{2}\right)$ is a genus $n$ Heegaard splitting of $S^{3}$, which is unique up to homeomorphism within $S^{3}$; we will denote this surface by $n\left(S^{3}, T^{2}\right)$.

In the special case of $\left(M_{1}, F_{1}\right) \#\left(S^{3}, F_{2}\right)=(M, F)$, note that $M_{1} \cong M$, so we may think of $M_{1}$ and $M$ as the same 3-manifold. Viewed this way, we see that $(M, F) \#\left(S^{3}, S^{2}\right)=(M, F)$ for any surface $(M, F)$. Another important connected sum involving $S^{3}$ is $(M, F) \#\left(S^{3}, T^{2}\right)$, which is called an order one stabilization of $(M, F)$. Any two order one stabilizations of $(M, F)$ are ambient isotopic in $M$, provided they involve the same component of $F$. A stabilization of $(M, F)$ is a surface obtained by a finite sequence of order one stabilizations on $(M, F)$. Two surfaces $\left(M, F_{1}\right)$ and $\left(M, F_{2}\right)$ in the same 3-manifold are stably isotopic if and only if there exist stabilizations of both surfaces which are ambient isotopic in $M$. Two surfaces $\left(M_{1}, F_{1}\right)$ and $\left(M_{2}, F_{2}\right)$ are stably equivalent if and only if there exists a homeomorphism $h: M_{1} \rightarrow M_{2}$ such that $\left(M_{2}, h\left(F_{1}\right)\right)$ and $\left(M_{2}, F_{2}\right)$ are stably isotopic. The reader is warned that the term "stably equivalent" is more traditionally used to refer to our notion of "stably isotopic."

Given any surface $(M, F)$, can it be expressed as a connected sum where neither factor is $\left(S^{3}, S^{2}\right)$ ? If it cannot, then $(M, F)$ is prime. By convention, $\left(S^{3}, S^{2}\right)$ is not considered to be a prime surface. It is natural to ask whether every surface can be represented as a connected sum of prime factors, and if so, whether there is any sense in which such a representation is unique.

Let $(M, F)$ be a surface. Define a set $\mathbf{S}$ of disjoint 2-spheres in Int $M$ to be a decomposition system (of spheres) for the surface if and only if for every $S \in \mathbf{S}, S$ separates $M$ and $S$ intersects $F$ transversely in a single simple closed curve. The elements of $\mathbf{S}$ are decomposition spheres for the surface. Let $\mathbf{P}$ be the set of all surfaces $(R, G)$ for which $R$ is the closure of a component of $M-\cup \mathbf{S}$, and $G=F \cap R$. Let $\mathbf{F}$ be the set of all surfaces $\left(M^{\prime}, F^{\prime}\right)$ obtained from $(R, G) \in \mathbf{P}$ by attaching copies of $\left(B^{3}, D^{2}\right)$ to all elements of $\mathbf{S}$ in $\partial R$. The elements of $\mathbf{F}$ are the factors of the decomposition (the notation $\mathbf{P}$ was motivated by "punctured factors"). Note that there is a clear one-to-one correspondence between elements of $\mathbf{P}$ and $\mathbf{F}$. We may also refer to $\mathbf{F}$ as $\mathbf{F}(\mathbf{S})$ or $\mathbf{F}(M, F ; \mathbf{S})$ in case of ambiguity (likewise for $\mathbf{P})$. It should be pointed out that we want to think of $(R, G) \in \mathbf{P}$ as actually being contained in $(M, F)$, and equaling the intersection of $(M, F)$ and $\left(M^{\prime}, F^{\prime}\right) \in \mathbf{F}$.

The point of all this is that if $\mathbf{S}$ is a decomposition system for $(M, F)$, then $(M, F)$ is a connected sum of the factors $\mathbf{F}$. Such a set of factors, or the expression of $(M, F)$ as a connected sum of such a set of factors, is a decomposition of $(M, F)$. If all of the factors are prime, then we have a prime decomposition, and $\mathbf{S}$ is a prime decomposition system. 
By a fairly standard argument using a complexity function on $(M, F)$, which in this case would involve the number of components of $F$ and $\partial F$, the genus of $F$, and the number of factors in any prime decomposition of $M$ as a 3manifold, it is easy to prove the following theorem.

Theorem 1.1. Every surface $(M, F)$ other than $\left(S^{3}, S^{2}\right)$ has a prime decomposition.

The question remains as to whether such decompositions are unique in any sense. Suzuki's result, mentioned above, is the following.

Theorem 1.2 (Suzuki, 1975). If a connected surface $\left(S^{3}, F\right)$ has two prime decompositions

$$
\begin{aligned}
\left(S^{3}, F\right) & =\left(S^{3}, F_{1}\right) \# \cdots \#\left(S^{3}, F_{m}\right) \# p\left(S^{3}, T^{2}\right) \\
& =\left(S^{3}, F_{1}^{\prime}\right) \# \cdots \#\left(S^{3}, F_{n}^{\prime}\right) \# q\left(S^{3}, T^{2}\right)
\end{aligned}
$$

with $\left(S^{3}, F_{i}\right),\left(S^{3}, F_{j}^{\prime}\right) \not\left(S^{3}, T^{2}\right)$, then $m=n$ and, after reordering if necessary, for every $i=1, \ldots, n$ we have that $\left(S^{3}, F_{i}\right)$ is stably isotopic to $\left(S^{3}, F_{i}^{\prime}\right)$.

In general, we will say that a surface $(M, F)$ has unique prime decompositions up to stable equivalence if the following holds. Let $\mathbf{S}$ and $\mathbf{S}^{\prime}$ be any two prime decomposition systems for the surface. Let $\left\{\left(M_{i}, F_{i}\right)\right\}_{i=1}^{m}$ and $\left\{\left(M_{i}^{\prime}, F_{i}^{\prime}\right)\right\}_{i=1}^{n}$ consist of those factors in $\mathbf{F}(\mathbf{S})$ and $\mathbf{F}\left(\mathbf{S}^{\prime}\right)$ respectively which are not homeomorphic to $\left(S^{3}, T^{2}\right)$. Then $m=n$ and, after reordering if necessary, for every $i=1, \ldots, n$ we have that $\left(M_{i}, F_{i}\right)$ is stably equivalent to $\left(M_{i}^{\prime}, F_{i}^{\prime}\right)$. Furthermore, in the category where $M$ is oriented, we would prefer to be able to claim that the homeomorphisms involved in the stable equivalences, $h_{i}: M_{i} \rightarrow M_{i}^{\prime}$, are orientation-preserving. Our primary concern here is the determination of which surfaces possess this property.

\section{THREE-MANIFOLD TRIADS}

A triple $\left(M ; B_{1}, B_{2}\right)$ is a 3 -manifold triad if $M$ is a compact 3-manifold, if $B_{1}, B_{2} \subset \partial M$ are disjoint (possibly empty) compact 2-manifolds, and if $\partial M-\left(B_{1} \cup B_{2}\right)$ is homeomorphic to $\partial B_{1} \times I$ with $\partial B_{1}=\partial B_{1} \times 0$ and $\partial B_{2}=$ $\partial B_{1} \times 1$. A triad may be viewed as a cobordism between compact 2-manifolds $B_{1}$ and $B_{2}$.

Recall that a Heegaard splitting of a closed 3-manifold may be viewed as a surface which separates the 3-manifold into two handlebodies. In order to generalize the notion of Heegaard splitting to triads, we must first generalize the notion of a handlebody. A compression body is a triad $\left(W ; \partial_{-} W, \partial_{+} W\right)$ with $\partial_{+} W \neq \varnothing$, which has a decomposition into a collar of $\partial_{+} W$ and 2-handles and 3 -handles attached to this collar, and which is furthermore required to have no 2 -sphere components in $\partial_{-} W$. The last requirement implies that $W$ is irreducible (all 2-spheres in $W$ bound 3-balls in $W$ ). Furthermore, for any compression body $W, \partial_{-} W$ is incompressible in $W$ (there is no disk in $W$ whose boundary is an essential simple closed curve in $\partial_{-} W$ ). Note that any handlebody is a compression body. We will usually refer to a compression body $\left(W ; \partial_{-} W, \partial_{+} W\right)$ simply as $W$.

By considering a dual to the collar and handle decomposition described above, one can obtain an equivalent definition for a compression body, to wit: 
$\left(W ; \partial_{-} W, \partial_{+} W\right)$ is a compression body if and only if $\partial_{-} W$ has no 2 -sphere components and $W$ has a decomposition into a collar of $\partial_{-} W$ and 0 -handles and 1-handles. It is due to this definition that compression bodies are also known as hollow handlebodies, and $\partial_{-} W$ and $\partial_{+} W$ are referred to as the interior boundary and exterior boundary of $W$ respectively.

An optimal handle decomposition for a compression body $W$ is one in which we minimize the number of 3-handles (or 0-handles). In particular, an optimal 2- and 3-handle decomposition for a connected compression body $W$ has one 3-handle if $\partial_{-} W=\varnothing$ and no 3-handle if $\partial_{-} W$ is nonempty. Likewise for 0 -handles. A complete disk system $D$ for a compression body $W$ is a disjoint union of properly embedded disks, which may be viewed as the cores of the 2-handles in some optimal 2- and 3-handle decomposition, with boundaries extended vertically to $\partial_{+} W$.

A Heegaard splitting of a triad $\left(M ; B_{1}, B_{2}\right)$ is a surface $(M, F)$ such that $F$ is disjoint from $B_{1}$ and $B_{2}$, and which separates $M$ into two compression bodies $W_{1}$ and $W_{2}$ such that $F$ is their common exterior boundary and $B_{i}$ is the interior boundary of $W_{i}$. Note that this is only possible if $B_{1}$ and $B_{2}$ have no 2-sphere components. If $\partial M=\varnothing$, this corresponds exactly with the notion of a Heegaard splitting of a closed 3-manifold. The following is a theorem that is proven in a manner completely analogous to the case for a closed 3-manifold.

Theorem 2.1. If a 3-manifold triad $\left(M ; B_{1}, B_{2}\right)$ has no 2-sphere components in $B_{1}$ or $B_{2}$, then it possesses a Heegaard splitting.

A classical result known as the Reidemeister-Singer Theorem states that any two Heegaard splittings of a closed 3-manifold are stably isotopic. This statement and its proof readily generalize to say the same thing about triads.

Theorem 2.2. Any two Heegaard splittings of a 3-manifold triad are stably isotopic.

Proofs of the classical statement may be found in [Re, Sin, Cr, and Sie]. Waldhausen states the above generalization without proof in [W], for the case of a triad $\left(M ; B_{1}, B_{2}\right)$ in which $B_{1}$ and $B_{2}$ are closed. A proof of the theorem as it is stated here may be found in [M]. It is this theorem which will allow us to conclude that the factors of our surface are stably equivalent, by obtaining those factors as Heegaard splittings of the same triads, which will themselves be factors of a triad corresponding to the original surface. So we need to discuss connected sum decompositions of triads.

The connected sum of two triads is defined by

$$
\left(M ; B_{1}, B_{2}\right) \#\left(M^{\prime} ; B_{1}^{\prime}, B_{2}^{\prime}\right)=\left(M \# M^{\prime} ; B_{1} \cup B_{1}^{\prime}, B_{2} \cup B_{2}^{\prime}\right),
$$

where $M \# M^{\prime}$ is the standard connected sum of two 3-manifolds obtained by removing one 3-ball from each of Int $M$ and Int $M^{\prime}$ and gluing the resulting boundaries together. Our definitions for decompositions of triads are very similar to those we made for surfaces. A decomposition system for a triad $\left(M ; B_{1}, B_{2}\right)$ is a set $\mathbf{S}$ of disjoint separating 2 -spheres in Int $M$. The set

$$
\mathbf{P}=\mathbf{P}(\mathbf{S})=\mathbf{P}\left(M ; B_{1} . B_{2} ; \mathbf{S}\right)
$$

consists of all triples $\left(R ; C_{1}, C_{2}\right)$ for which $R$ is the closure of a component of $M-\cup \mathbf{S}$, and $C_{i}=R \cap B_{i}$. These are not triads due to the spheres of $\mathbf{S}$ 
in $\partial R$, but note that $\partial C_{1} \times I \subset \partial R$. The set of factors $\mathbf{F}$ is obtained from $\mathbf{P}$ by capping off 2 -spheres of $\mathbf{S}$ in $\partial R$ with 3-balls for every $\left(R ; C_{1}, C_{2}\right) \in \mathbf{P}$. The factors are 3-manifold triads, and $\left(M ; B_{1}, B_{2}\right)$ can be obtained as a connected sum of the factors. Note that a factor $\left(M^{\prime} ; C_{1}, C_{2}\right)$ which corresponds to $\left(R ; C_{1}, C_{2}\right) \in \mathbf{P}$ has the same 2-manifolds $C_{1}$ and $C_{2}$ in the triple. $\mathrm{A}$ decomposition is nontrivial if and only if none of the factors are $\left(S^{3} ; \varnothing, \varnothing\right)$. A triad is prime if it has no nontrivial decompositions. A prime decomposition system is one all of whose factors are prime. It follows immediately that a triad $\left(M ; B_{1}, B_{2}\right)$ is prime if and only if $M$ is prime as a 3-manifold, that a decomposition of a triad $\left(M ; B_{1}, B_{2}\right)$ is a prime decomposition if and only if it provides a prime decomposition of $M$ as a 3-manifold, and that every triad has a prime decomposition.

Likewise, uniqueness of prime decompositions of 3-manifold triads follows from uniqueness of prime decompositions of 3-manifolds, but we need to strengthen the usual statement of uniqueness with the following theorem. As with 3-manifolds, we require that if $M$ is nonorientable, then the prime decompositions under consideration must have no factors of $S^{2} \times S^{1}$ (the choice of decomposition spheres may be changed so as to replace a factor of $S^{2} \times S^{1}$ with a nonorientable 2-sphere bundle over $S^{1}$ ).

Theorem 2.3. Let $\left(M ; B_{1}, B_{2}\right)$ be a triad and let $\mathbf{S}$ and $\mathbf{S}^{\prime}$ be prime decomposition systems of this triad with associated sets of factors $\mathbf{F}=\left\{\left(M_{i} ; C_{i 1}, C_{i 2}\right)\right\}_{i=1}^{m}$ and $\mathbf{F}^{\prime}=\left\{\left(M_{i}^{\prime} ; C_{i 1}^{\prime}, C_{i 2}^{\prime}\right)\right\}_{i=1}^{n}$, and satisfying the property that if $M$ is nonorientable, then no factor is $\left(S^{2} \times S^{1} ; \varnothing, \varnothing\right)$. Then $m=n$ and, after reordering, for every $i=1, \ldots, n$ :

(1) $\partial M_{i}=M_{i} \cap \partial M=M_{i}^{\prime} \cap \partial M=\partial M_{i}^{\prime}$,

(2) there exists a homeomorphism $h_{i}:\left(M_{i} ; C_{i 1}, C_{i 2}\right) \rightarrow\left(M_{i}^{\prime} ; C_{i 1}^{\prime}, C_{i 2}^{\prime}\right)$,

(3) $h_{i} \mid \partial M_{i}$ is the identity (thus note $C_{i 1}=C_{i 1}^{\prime}$ and $C_{i 2}=C_{i 2}^{\prime}$ ), and

(4) if $M$ is oriented, then $h_{i}$ is orientation-preserving.

Proof. All of this follows from considering $\mathbf{S}$ and $\mathbf{S}^{\prime}$ as providing prime decompositions of $M$ as a 3-manifold, except that conclusions (1) and (3) are not usually included in the statement of the theorem. In order to see that these conclusions are valid, consider a proof such as that in [He, Chapter 3]. Let $\mathbf{P}$ and $\mathbf{P}^{\prime}$ correspond to $\mathbf{F}$ and $\mathbf{F}^{\prime}$, with, for example, $\left(R_{i} ; C_{i 1}, C_{i 2}\right) \in$ $\mathbf{P}$ corresponding to $\left(M_{i} ; C_{i 1}, C_{i 2}\right) \in \mathbf{F}$. One constructs a sequence $\mathbf{S}=$ $\mathbf{S}_{0}, \mathbf{S}_{1}, \ldots, \mathbf{S}_{n}=\mathbf{S}^{\prime}$ of prime decomposition systems where each $\mathbf{S}_{k+1}$ is obtained from $\mathbf{S}_{k}$ by replacing one 2-sphere with another, causing an exchange between two $R_{i}, R_{j} \in \mathbf{P}_{k}$ of a punctured 3-sphere not meeting $\partial M$. This provides a sequence from $\left(R_{m} ; C_{m 1}, C_{m 2}\right) \in \mathbf{P}$ to $\left(R_{n}^{\prime} ; C_{n 1}^{\prime}, C_{n 2}^{\prime}\right) \in \mathbf{P}^{\prime}$ (renumbering if necessary) through additions or subtractions of punctured 3-spheres not affecting the boundary, and thus provides an (orientation-preserving) homeomorphism between $\left(M_{m} ; C_{m 1}, C_{m 2}\right)$ and $\left(M_{n}^{\prime} ; C_{n 1}^{\prime}, C_{n 2}^{\prime}\right)$ which is the identity on their boundary.

Our plan is to consider prime decompositions of a surface, show that these may be viewed as decompositions of a certain 3-manifold triad, and that they will in fact be prime decompositions of this triad. In doing this we will need the following lemma, which is stated here essentially as it appears in [CG, Lemma 1.1 , and which is a generalization of a result in [Ha] (an account of whose proof 
is also given in [ $\mathrm{Ja}$, Chapter 2]). A proof of a version of this lemma appears in [BoO].

Lemma 2.4. Let $(M, F)$ be a Heegaard splitting of $\left(M ; B_{1}, B_{2}\right)$. Let $S$ be a disjoint union of essential 2-spheres and disks properly embedded in $M$ with $\partial S \subset\left(B_{1} \cup B_{2}\right)$. Then there exists a similar disjoint union of essential 2-spheres and disks $S^{\prime}$ in $M$ such that

(1) there exists a sequence $S=S_{0}, S_{1}, \ldots, S_{n}=S^{\prime}$ where $S_{i+1}$ is obtained from $S_{i}$ either by ambient isotopy or by surgery along a disk $D$ with $\partial D \subset S_{i}$, Int $D \cap S_{i}=\varnothing$, and $D \cap F=\varnothing$,

(2) each component of $S^{\prime}$ meets $F$ in a single simple closed curve, and

(3) there exist complete disk systems $D_{1}$ and $D_{2}$ for the two compression bodies on either side of $F$ such that $D_{1} \cap S^{\prime}=D_{2} \cap S^{\prime}=\varnothing$.

Furthermore, if $M$ is irreducible (and therefore $S$ consists of disks), then $S^{\prime}$ is ambient isotopic to $S$.

\section{The MAXIMAL TRIAD OF A SURFACE}

In the previous section, we mentioned that there will be a certain 3-manifold triad associated with the surface. We are now ready to define this object, which will be called the maximal triad of the surface. We will only define the maximal triad (and will only need to use it) for surfaces $(M, F)$ for which the 3-manifold $M-F$ is irreducible.

Let $(M, F)$ be a surface for which $M-F$ is irreducible. A triad $\left(T ; B_{1}, B_{2}\right)$ is a maximal triad for $(M, F)$ if and only if the following conditions are satisfied:

(1) $T \subset M$,

(2) the components of $B_{1}$ and $B_{2}$ are either properly embedded in $M$ or lie in $\partial M, \partial T-\left(B_{1} \cup B_{2}\right)$ is contained in $\partial M$, and no component of $B_{1} \cup B_{2}$ is parallel to $\partial M$ in $M-F$,

(3) no component of $B_{1} \cup B_{2}$ is a 2-sphere,

(4) $B_{1} \cup B_{2}$ is incompressible in the closure of $M-T$, and

(5) $F$ is a Heegaard splitting of $\left(T ; B_{1}, B_{2}\right)$.

Note that if $M$ is oriented, then it induces a canonical orientation on $T$. We immediately set about proving existence and uniqueness of maximal triads.

Theorem 3.1. Every surface $(M, F)$ for which $M-F$ is irreducible possesses a maximal triad.

Proof. Since $F$ is two-sided in $M$, let $M^{\prime} \cong F \times[-1,1]$ be a bicollar of $F$ in $M$, and let $B_{1}^{\prime}=F \times 1$ and $B_{2}^{\prime}=F \times-1$. If either $B_{1}^{\prime}$ or $B_{2}^{\prime}$ has any component parallel to $\partial M$ in $M-F$, then thicken the bicollar to the boundary at that place. We now have a triad $\left(M^{\prime} ; B_{1}^{\prime}, B_{2}^{\prime}\right)$ which satisfies conditions (1), (2), and (5). Let $N^{\prime}$ be the closure of $M-M^{\prime}$.

If either $B_{1}^{\prime}$ or $B_{2}^{\prime}$ is compressible into $N^{\prime}$, then add a 2-handle to $M^{\prime}$ with core a compressing disk. $F$ remains a Heegaard splitting since on each side of $F$ there is a collar of $F$ with 2-handles attached to this collar. Continue adding such 2-handles until there are no more such compressing disks; the process must terminate since the addition of each 2-handle reduces the genus of $B_{1}^{\prime}$ or $B_{2}^{\prime}$. At this stage we have a triad $\left(M^{\prime \prime} ; B_{1}^{\prime \prime}, B_{2}^{\prime \prime}\right)$ which satisfies every condition except (3). Of course, if during the additions of the 2-handles any component of $B_{1}^{\prime \prime}$ 
or $B_{2}^{\prime \prime}$ becomes parallel to $\partial M$ in $M-F$, then $M^{\prime \prime}$ should be thickened to the boundary at that place. Let $N^{\prime \prime}$ be the closure of $M-M^{\prime \prime}$.

Now if any component $S$ of $B_{1}^{\prime \prime} \cup B_{2}^{\prime \prime}$ is a 2-sphere, then irreducibility of $M-F$ implies that $S$ bounds a 3-ball $B$ in $M-F$, and necessarily $B \subset N^{\prime \prime}$. So add $B$ to $M^{\prime \prime}$ and remove $S$ from $B_{1}^{\prime \prime}$ or $B_{2}^{\prime \prime}$. Do this with all 2-sphere components of $B_{1}^{\prime \prime} \cup B_{2}^{\prime \prime}$. The result is a triad $\left(T ; B_{1}, B_{2}\right)$ which satisfies all five conditions, and is therefore a maximal triad for the surface.

In order to prove uniqueness, we rely on the work of [Bo, $\S 2]$, in which Bonahon proves that for $M$ irreducible, $\partial M$ possesses a maximal compression body $W \subset M$ with $\partial_{+} W=\partial M$, which is unique up to ambient isotopy of $M$ with $\partial M$ fixed. This work immediately generalizes to prove, for any compact 2-manifold $F \subset \partial M$, that a maximal compression body in $M$ whose exterior boundary is $F$ is unique up to ambient isotopy in $M$ with $F$ fixed. The basic idea of his proof is that a system of disks with boundary in $F$, and therefore compression bodies with exterior boundary $F$, can be isotoped into the interior of a maximal compression body, and that two maximal compression bodies, one inside the other, have parallel interior boundaries.

In order to be consistent about the numbering, we suppose that for a given surface $F$ we can refer to the two "sides" of $F$ as side 1 and side 2 . In other words, for a sufficiently thin bicollar $F \times[-1,1]$ (one contained in every maximal triad of $F$ under consideration) refer to $F \times 1$ as side 1 , and $F \times-1$ as side 2 . Then for any maximal triad $\left(T ; B_{1}, B_{2}\right)$ for $F$, require that the compression body with $B_{1}$ as interior boundary must contain side 1 , and the compression body with $B_{2}$ as interior boundary must contain side 2 .

Theorem 3.2. Let $(M, F)$ be a surface with $M-F$ irreducible. Then any two maximal triads for $(M, F)$ are unique up to an ambient isotopy of $M$ with $F$ fixed.

Proof. Let $\left(T ; B_{1}, B_{2}\right)$ and $\left(T^{\prime} ; B_{1}^{\prime}, B_{2}^{\prime}\right)$ be two maximal triads for $(M, F)$. Let $C \cong F \times[-1,1]$ be a bicollar for $F$ in $M$, which is contained in both maximal triads. Let $N$ be the closure of $M-C$, and note that $N$ is irreducible. Let $G$ be the union of $F \times 1$ and $F \times-1$, and note that $G \subset \partial N$. Let $W$ and $W^{\prime}$ be the closures of $T-C$ and $T^{\prime}-C$ respectively. Then $W$ and $W^{\prime}$ are maximal compression bodies for $G$ in $N$, and by [Bo] they must be ambient isotopic in $N$ with $G$ fixed. This isotopy easily extends to an ambient isotopy of $M$ with $F$ fixed, which takes one maximal triad to the other.

Our main reason for studying maximal triads of surfaces is the fact that they provide information on whether two surfaces are stably isotopic. This is accomplished by the following theorem.

Theorem 3.3. Let $(M, F)$ and $\left(M, F^{\prime}\right)$ be two surfaces in the same 3-manifold such that $M-F$ and $M-F^{\prime}$ are irreducible. $(M, F)$ and $\left(M, F^{\prime}\right)$ are stably isotopic if and only if they have the same maximal triad (up to ambient isotopy in $M$ ).

Proof. If they have the same maximal triad, then they are both Heegaard splittings of this triad, and so they are stably isotopic by the Reidemeister-Singer Theorem (Theorem 2.2). So now suppose that $(M, F)$ and $\left(M, F^{\prime}\right)$ are stably isotopic. Then there exists a surface $\left(M, F^{\prime \prime}\right)$ which is a stabilization of both. 
Construct a maximal triad for $(M, F)$ in the following way. Let $C_{1}, \ldots, C_{n}$ be disjoint 3-balls in $M$ which we remove to perform the connected sum of $n$ copies of $\left(S^{3}, T^{2}\right)$ in order to stabilize $(M, F)$ to $\left(M, F^{\prime \prime}\right)$. So we replace each disk of $C_{i} \cap F$ with an unknotted punctured torus inside $C_{i}$. Since these punctured tori compress on both sides, we can start building a maximal triad for $\left(M, F^{\prime \prime}\right)$ by taking a bicollar and adding 2 -handles with cores these compressing disks, in such a way that these 2-handles fill in each of the 3-balls $C_{i}$ entirely. The resulting 3-manifold at this stage is then a bicollar of $F$, since it consists of each 3-ball $C_{i}$ and a bicollar of $F^{\prime \prime}-\bigcup_{i=1}^{n} C_{i}=F-\bigcup_{i=1}^{n} C_{i}$. Now continue to build a maximal triad by adding 2-handles and 3-handles as in the proof of the theorem above. The result is a triad $\left(T ; B_{1}, B_{2}\right)$ which is a maximal triad for both $(M, F)$ and $\left(M, F^{\prime \prime}\right)$.

Likewise build a triad $\left(T^{\prime} ; B_{1}^{\prime}, B_{2}^{\prime}\right)$ which is a maximal triad for both $\left(M, F^{\prime}\right)$ and $\left(M, F^{\prime \prime}\right)$. Now $M-F^{\prime \prime}$ is irreducible, and by the previous theorem we can perform an ambient isotopy in $M$ (with $F^{\prime \prime}$ fixed) taking the maximal triad $\left(T^{\prime} ; B_{1}^{\prime}, B_{2}^{\prime}\right)$ of $\left(M, F^{\prime \prime}\right)$ to the maximal triad $\left(T ; B_{1}, B_{2}\right)$ of $\left(M, F^{\prime \prime}\right)$. We therefore have an ambient isotopy of $M$ between maximal triads for $(M, F)$ and $\left(M, F^{\prime}\right)$.

\section{UNIQUENESS OF PRIME DECOMPOSITIONS}

We are now ready to use maximal triads to help us prove theorems on uniqueness of prime decompositions of surfaces. Our first task is to show how a decomposition of a surface gives rise to a decomposition of its maximal triad.

Lemma 4.1. Let $(M, F)$ be a surface such that $M-F$ is irreducible, and let $\mathbf{S}$ be a decomposition system for the surface. Then we may assume without loss of generality that $\mathbf{S}$ is contained in the interior of the maximal triad of $(M, F)$.

Proof. Each sphere of $\mathbf{S}$ meets the interior of $F$ in a single simple closed curve. Choose a bicollar of $F$ in $M$ such that the spheres of $\mathbf{S}$ meet this bicollar in vertical annuli. For each sphere $S$ in $\mathbf{S}$, this bicollar separates $S$ into two disks; add two 2-handles to the bicollar whose cores are these disks. When this is done we have a triad containing $\mathbf{S}$ in its interior. Now add 2-handles and 3-handles to complete this triad to a maximal triad for the surface.

Note also in the previous lemma that since the spheres of $\mathbf{S}$ separate $M$, they must also separate the maximal triad. Thus if $F$ is connected, and therefore its maximal triad is connected, then $\mathbf{S}$ is a decomposition system for the maximal triad. Now for every $\left(Q ; C_{1}, C_{2}\right) \in \mathbf{P}\left(T ; B_{1}, B_{2} ; \mathbf{S}\right)$ there exists a unique $(R, G) \in \mathbf{P}(M, F ; \mathbf{S})$ such that $Q \subset R$. Then we may view the copies of $\left(B^{3}, D^{2}\right)$ with which we cap off members of $\mathbf{P}(M, F ; \mathbf{S})$ to obtain the factors $\mathbf{F}(M, F ; \mathbf{S})$ as providing the 3-balls with which we cap off members of $\mathbf{P}\left(T ; B_{1}, B_{2} ; \mathbf{S}\right)$ to obtain the factors $\mathbf{F}\left(T ; B_{1}, B_{2} ; \mathbf{S}\right)$. Thus there is a one-to-one correspondence between factors $\left(T^{\prime} ; C_{1}, C_{2}\right)$ of the maximal triad and factors $\left(M^{\prime}, F^{\prime}\right)$ of the surface such that $F^{\prime} \subset T^{\prime} \subset M^{\prime}$. We now need to conclude that these corresponding factors are related in the way that one would hope.

Theorem 4.2. Let $(M, F)$ be a connected surface such that $M-F$ is irreducible, let $\left(T ; B_{1}, B_{2}\right)$ be the maximal triad for this surface, and let $\mathbf{S}$ be a decomposition system for the surface which is contained in the interior of the maximal 
triad. If $\left(M^{\prime}, F^{\prime}\right)$ and $\left(T^{\prime} ; C_{1}, C_{2}\right)$ are corresponding factors of the surface and the maximal triad, then $\left(T^{\prime} ; C_{1}, C_{2}\right)$ is the maximal triad for $\left(M^{\prime}, F^{\prime}\right)$.

Proof. Let $(R, G) \in \mathbf{P}(M, F ; \mathbf{S})$ correspond to $\left(M^{\prime}, F^{\prime}\right)$, and $\left(Q ; C_{1}, C_{2}\right) \in$ $\mathbf{P}\left(T ; B_{1}, B_{2} ; \mathbf{S}\right)$ correspond to $\left(T^{\prime} ; C_{1}, C_{2}\right)$. In the proof of the previous lemma, we built the maximal triad for $(M, F)$ by first taking a bicollar of $F$ and adding 2-handles containing disks in the spheres of $\mathbf{S}$ as cores. $\left(Q ; C_{1}, C_{2}\right)$ is obtained by taking the intersection of this object with $R$ (which provides a bicollar of $G$ plus 2-handles lying on the 2-sphere boundary components), and then adding 2-handles and 3-handles that lie within $R$. In the copies of $B^{3}$ which capped off $R$, we also take a bicollar of $D^{2}$, thus having a bicollar of the entire 2-manifold $F^{\prime}$, and then add the two 3-handles in $B^{3}$ which meet this bicollar and the two 2-handles lying along the boundary. This will be a maximal triad for $\left(M^{\prime}, F^{\prime}\right)$, and is in fact $\left(T^{\prime} ; C_{1}, C_{2}\right)$.

We now turn our attention to prime decompositions. We would like to conclude that a prime decomposition of the surface corresponds to a prime decomposition of its maximal triad, but we need to make an exception for certain 3-manifolds which contain nonseparating 2-spheres.

Theorem 4.3. Let $(M, F)$ be a prime, connected surface such that $M-F$ is irreducible and such that no nonseparating 2-sphere in $M$ intersects $F$ in a single simple closed curve. Let $\left(T ; B_{1}, B_{2}\right)$ be the maximal triad for $(M, F)$. Then either $\left(T ; B_{1}, B_{2}\right)$ is prime, or $(M, F)=\left(S^{3}, T^{2}\right)$ and $T=S^{3}$.

Corollary 4.4. Let $(M, F)$ be a connected surface such that $M-F$ is irreducible and such that no nonseparating 2-sphere in $M$ intersects $F$ in a single simple closed curve. Then any prime decomposition of $(M, F)$ corresponds to a decomposition of its maximal triad into factors each of which is either prime or trivial.

Note that the condition on nonseparating 2-spheres is certainly satisfied if $M$ contains no nonseparating 2-spheres at all. For $M$ orientable, this is equivalent to saying that $M$ has no factor of $S^{2} \times S^{1}$.

Proof of Theorem. $(M, F)$ is a Heegaard splitting of $\left(T ; B_{1}, B_{2}\right)$. Thus if $T=S^{3}$, then $(M, F)$ is a Heegaard splitting $n\left(S^{3}, T^{2}\right)$ of $S^{3}$, and the fact that it is prime implies that $n=1$.

Now suppose that $T \neq S^{3}$, and assume that $\left(T ; B_{1}, B_{2}\right)$ is not prime. Then there exists a nontrivial decomposition sphere $S$ for this triad. By Lemma 2.4 there exists an essential 2-sphere $S^{\prime}$ in $T$ which meets $F$ in a single simple closed curve. $S^{\prime}$ must separate $M$ since $M$ contains no nonseparating 2sphere which meets $F$ in a single simple closed curve. Since $(M, F)$ is prime, $S^{\prime}$ induces a decomposition

$$
(M, F)=\left(M^{\prime}, F^{\prime}\right) \#\left(S^{3}, S^{2}\right) .
$$

But this implies that $S^{\prime}$ bounds a 3-ball in $M$ and thus in $T$, contradicting the fact that it is supposed to be essential. Thus there can exist no such nontrivial decomposition sphere $S$ for the triad, and therefore $\left(T ; B_{1}, B_{2}\right)$ must be prime.

Our condition on nonseparating 2-spheres was necessary to prevent the possibility of a 2-sphere which separates the maximal triad $\left(T ; B_{1}, B_{2}\right)$ but which 

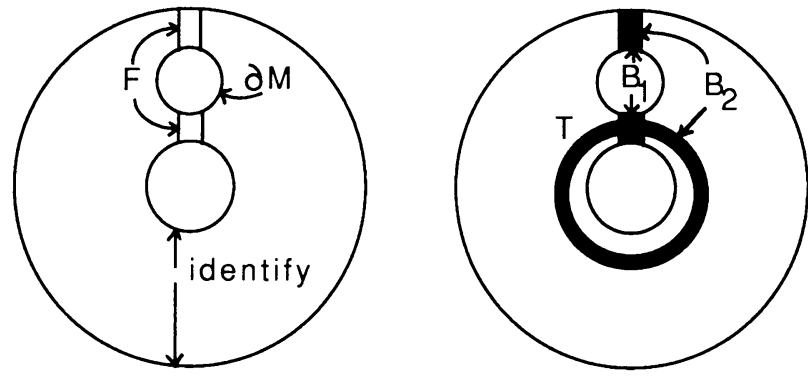

FIGURE 1

does not separate the 3-manifold $M$. Is this in fact possible? The answer is yes, as the following example shows. Let $M$ be $S^{2} \times S^{1}$ with the interior of a 3-ball removed. Let $F$ be an annulus properly embedded in $M$ such that a nonseparating 2-sphere of $M$ meets $F$ transversely in a single simple closed curve (see Figure 1). Now any 2-sphere in $M$ which intersects $F$ in a single simple closed curve is either nonseparating or induces a trivial decomposition, and therefore $(M, F)$ is prime. The maximal triad $\left(T ; B_{1}, B_{2}\right)$ is obtained by adding a single 2-handle on each side of $F$, and on one side thickening to the boundary of $M . B_{1}$ will consist of two disks on $\partial M$, and $B_{2}$ will consist of two disks properly embedded in $M$. The cores of the two 2-handles join to give a 2-sphere which is nonseparating in $M$ but does separate $T$, and induces a decomposition of the triad both of whose factors are copies of $\left(D^{2} \times I ; D^{2} \times 0, D^{2} \times 1\right)$. Therefore the maximal triad of this prime surface is not prime.

We are now ready to prove that a surface $(M, F)$ has unique prime decompositions up to stable equivalence, provided that no nonseparating 2-sphere of $M$ is disjoint from $F$ or meets it in a single simple closed curve. We will accomplish this in stages, first for $M-F$ irreducible and $F$ connected, then for $F$ not connected, and finally for $M-F$ reducible. We start with $F$ connected because it is easier to follow what is happening in this situation.

Theorem 4.5. Let $(M, F)$ be a connected surface such that $M-F$ is irreducible and no nonseparating 2-sphere of $M$ meets $F$ in a single simple closed curve. Then $(M, F)$ has unique prime decompositions up to stable equivalence.

Proof. Let $\mathbf{S}$ and $\mathbf{S}^{\prime}$ be two prime decomposition systems for $(M, F)$, and assume without loss of generality that both are contained in the maximal triad $\left(T ; B_{1}, B_{2}\right)$ of $(M, F)$. Let $\left\{\left(M_{i}, F_{i}\right)\right\}_{i=1}^{m}$ and $\left\{\left(M_{i}^{\prime}, F_{i}^{\prime}\right)\right\}_{i=1}^{n}$ consist of those factors in $\mathbf{F}(M, F ; \mathbf{S})$ and $\mathbf{F}\left(M, F ; \mathbf{S}^{\prime}\right)$ respectively which are not $\left(S^{3}, T^{2}\right)$. Let $\left\{\left(T_{i} ; C_{i 1}, C_{i 2}\right)\right\}_{i=1}^{m}$ and $\left\{\left(T_{i}^{\prime}, C_{i 1}^{\prime}, C_{i 2}^{\prime}\right)\right\}_{i=1}^{n}$ be the corresponding factors in $\mathbf{F}\left(T ; B_{1}, B_{2} ; \mathbf{S}\right)$ and $\mathbf{F}\left(T ; B_{1}, B_{2} ; \mathbf{S}^{\prime}\right)$, which in each case are exactly those factors of the maximal triad that are prime. Since all of the other factors are trivial, the factors of the maximal triad listed above provide two prime decompositions of the maximal triad. Note that no factor of the triad is $\left(S^{2} \times\right.$ $\left.S^{1} ; \varnothing, \varnothing\right)$ since the fact that some factor of the surface would have to be a Heegaard splitting of this triad factor (necessarily of genus 1 since it is prime and by [W] every Heegaard splitting of $S^{2} \times S^{1}$ is a stabilization of the standard genus 1 splitting) would imply that there exists a nonseparating 2 -sphere in $M$ 
which meets $F$ in a single simple closed curve. Therefore by Theorem 2.3, $m=n$ and, after reordering, for every $i=1, \ldots, n$ :

(1) $\partial T_{i}=\partial T_{i}^{\prime}$

(2) there exists a homeomorphism $h_{i}:\left(T_{i} ; C_{i 1}, C_{i 2}\right) \rightarrow\left(T_{i}^{\prime} ; C_{i 1}^{\prime}, C_{i 2}^{\prime}\right)$,

(3) $h_{i} \mid \partial T_{i}$ is the identity, $C_{i 1}=C_{i 1}^{\prime}, C_{i 2}=C_{i 2}^{\prime}$, and

(4) if $M$ is oriented, then $h_{i}$ is orientation-preserving.

Assume that the reordering is also performed on the factors of the surface to maintain their correspondence with the factors of the triad. Note that we now have $m=n$.

For every $i=1, \ldots, n$, the fact that $\partial T_{i}=\partial T_{i}^{\prime}$ implies that $M_{i}-T_{i}$ and $M_{i}^{\prime}-T_{i}^{\prime}$ must consist of the same components of $M-T$ (if they are nonempty), and therefore these complements are equal. Since $h_{i} \mid \partial T_{i}$ is the identity, $h_{i}$ extends to a homeomorphism $h_{i}: M_{i} \rightarrow M_{i}^{\prime}$ which is the identity on $M_{i}-T_{i}$. Note that $h_{i} \mid \partial M_{i}$ is the identity, and $h_{i}$ is orientation-preserving if $M$ is oriented. Since $\left(M_{i}^{\prime}, h_{i}\left(F_{i}\right)\right)$ and $\left(M_{i}^{\prime}, F_{i}^{\prime}\right)$ have the same maximal triad $\left(T_{i}^{\prime}=h_{i}\left(T_{i}\right) ; C_{i 1}, C_{i 2}\right)$, it follows from Theorem 3.3 that they are stably isotopic, and therefore that $\left(M_{i}, F_{i}\right)$ and $\left(M_{i}^{\prime}, F_{i}^{\prime}\right)$ are stably equivalent.

Note that Suzuki's result, Thecrem 1.2, is a corollary to this theorem (an orientation-preserving homeomorphism of $S^{3}$ can be realized as the end result of an ambient isotopy of $S^{3}$ ). We now drop the condition that $F$ be connected. The proof of the following theorem is just a generalization of the proof of the preceding theorem.

Theorem 4.6. Let $(M, F)$ be a surface such that $M-F$ is irreducible and no nonseparating 2-sphere of $M$ meets $F$ in a single simple closed curve. Then $(M, F)$ has unique prime decompositions up to stable equivalence.

Proof. As in the previous proof, let $\mathbf{S}$ and $\mathbf{S}^{\prime}$ be two prime decomposition systems for $(M, F)$, assume without loss of generality that both are contained in the maximal triad $\left(T ; B_{1}, B_{2}\right)$ of $(M, F)$, and let $\left\{\left(M_{i}, F_{i}\right)\right\}_{i=1}^{m}$ and $\left\{\left(M_{i}^{\prime}, F_{i}^{\prime}\right)\right\}_{i=1}^{n}$ be the factors of the surface which are not $\left(S^{3}, T^{2}\right)$. Now let $T_{1}, \ldots, T_{q}$ be the components of $T$, one for each component of $F$, and let $\left\{T_{i j}\right\}$ and $\left\{T_{i j}^{\prime}\right\}$ be the nontrivial factors of $T_{i}$ under those spheres of $\mathbf{S}$ and $\mathbf{S}^{\prime}$ which meet $T_{i}$. By uniqueness of prime decompositions of each $\operatorname{triad} T_{i}$, there is a one-to-one correspondence $T_{i j} \leftrightarrow T_{i j}^{\prime}$ (after reordering the $j$ 's) such that for every $i$ and $j$ there exists an (orientation-preserving) homeomorphism

$$
h_{i j}:\left(T_{i j} ; C_{i j 1}, C_{i j 2}\right) \rightarrow\left(T_{i j}^{\prime} ; C_{i j 1}, C_{i j 2}\right)
$$

which is the identity on $\partial T_{i j}$.

Consider a graph $\Gamma$ whose vertices consist of triad factors $T_{i j}$ and of components of $M-T$, and whose edges join any two vertices of different types which meet along their boundary. Similarly construct $\Gamma^{\prime}$ using $T_{i j}^{\prime}$. Since any two vertices joined by an edge must be in the same factor of the surface, there is a one-to-one correspondence between components of $\Gamma$ (or $\Gamma^{\prime}$ ) and factors of the surface other than copies of $\left(S^{3}, T^{2}\right)$, where for each factor $\left(M_{k}, F_{k}\right)$, the corresponding component $\Gamma_{k}$ of $\Gamma$ consists of all vertices contained in $M_{k}$ and the edges joining them.

Let $\left(M_{k}, F_{k}\right)$ be a factor of the surface other than a copy of $\left(S^{3}, T^{2}\right)$, with corresponding component $\Gamma_{k}$ of $\Gamma$. Let $\sigma: \Gamma_{k} \rightarrow \Gamma^{\prime}$ map vertices $T_{i j}$ to the 
corresponding $T_{i j}^{\prime}$, components of $M-T$ to themselves, and edges joining two of these vertices to the edge in $\Gamma^{\prime}$ corresponding to the same boundary along which the vertices meet; this latter edge must exist since the maps $h_{i j} \mid \partial T_{i j}$ are identities. Clearly $\sigma\left(\Gamma_{k}\right)$ is connected. In fact, $\sigma\left(\Gamma_{k}\right)$ must be a component of $\Gamma^{\prime}$ since any vertex of $\Gamma^{\prime}$ connected to $\sigma\left(\Gamma_{k}\right)$ by an edge must correspond to a vertex in $\Gamma$ connected to $\Gamma_{k}$ by an edge. Renumber the factors of the surface under $\mathbf{S}^{\prime}$ and the corresponding components of $\Gamma^{\prime}$ so that $\sigma\left(\Gamma_{k}\right)=\Gamma_{k}^{\prime}$ for all $k$. Thus $\sigma$ induces a one-to-one correspondence between components of $\Gamma$ and $\Gamma^{\prime}$.

We now have $m=n$. For every $k$, the homeomorphisms $h_{i j}$ on vertices $T_{i j}$ of $\Gamma_{k}$ extend as the identity across components of $M-T$ which are vertices of $\Gamma_{k}$, to produce an (orientation-preserving) homeomorphism $h_{k}: M_{k} \rightarrow M_{k}^{\prime}$ such that $h_{k}$ maps the maximal triad of $\left(M_{k}, F_{k}\right)$ onto that of $\left(M_{k}^{\prime}, F_{k}^{\prime}\right)$. By Theorem 3.3, $\left(M_{k}, F_{k}\right)$ and $\left(M_{k}^{\prime}, F_{k}^{\prime}\right)$ are stably equivalent.

We are now ready to drop the condition that $M-F$ be irreducible. The 2-spheres in $M-F$ which do not bound 3-balls will give rise to specific factors of the surface which must correspond between different prime decompositions, and after these are removed what remains will be surfaces with irreducible complements. In order to obtain the relationship between a 2-sphere in the complement and a factor of the surface, we use the following lemma.

Lemma 4.7. Let $(M, F)$ be a prime surface and $T \subset(M-F)$ a separating 2-sphere which does not bound a 3-ball in $M-F$. Then either

(1) $M$ is a prime 3-manifold, $F$ is a 2-sphere, and $T$ bounds a 3-ball in $M$ containing $F$, or

(2) $M=S^{3}, F$ is a disjoint union of two 2-spheres, and $T$ separates the components of $F$.

Proof. There is a component of $F$ on one side or the other of $T$. Let $\alpha$ be an arc in $M$ with one endpoint on $T$ and the other endpoint on $F$, and meeting neither of these in its interior. Let $S$ be the 2-sphere boundary component of a small regular neighborhood of $T \cup \alpha$ which meets $F$ in a single simple closed curve. Note that $S$ separates $M$ since $T$ does. Since $(M, F)$ is prime, $S$ must bound a copy of $\left(B^{3}, D^{2}\right)$ on one side or the other. It cannot be the side containing $T$ and $\alpha$, since in that case $T$ would bound a 3-ball in $M-F$. Therefore $F$ is the union of the disk in $\left(B^{3}, D^{2}\right)$ and the disk in the regular neighborhood of $\alpha$, and is therefore a 2-sphere. Furthermore $F$ is contained in the union of the 3-ball of $\left(B^{3}, D^{2}\right)$ and the regular neighborhood of $\alpha$, which is a 3-ball bounded by $T$ in $M$.

Since the same procedure can be used on the other side of $T$ if there are components of $F$ on that side, it follows that if $T$ separates components of $F$, then $M=S^{3}$ and $F$ consists of two disjoint 2-spheres, one on each side of $T$.

So suppose that $T$ does not separate components of $F$. Then $F$ is a single 2-sphere which bounds a 3-ball in $M$. If $M$ were not a prime 3-manifold, then one could choose a separating 2 -sphere $S$ in $M$ which induces a nontrivial decomposition of $M$ as a 3-manifold, and without loss of generality $S$ can be taken to meet $F$ in a single simple closed curve. But this $S$ would then yield a nontrivial decomposition of the surface $(M, F)$, contradicting the fact that it is prime. Therefore, there is no such $S$, and $M$ must be a prime 3-manifold. 
Theorem 4.8. Let $(M, F)$ be a surface such that no nonseparating 2-sphere of $M$ is disjoint from $F$ or meets it in a single simple closed curve. Then $(M, F)$ has unique prime decompositions up to stable equivalence.

Proof. Let $\mathbf{S}$ and $\mathbf{S}^{\prime}$ be two prime decomposition systems for $(M, F)$. Let $N$ be the 3-manifold obtained by cutting $M$ along $F$, or in other words, if $F \times[-1,1]$ is a bicollar of $F$ in $M$ which meets the spheres of $\mathbf{S}$ and $\mathbf{S}^{\prime}$ in vertical annuli, then $N$ is the submanifold of $M$ obtained by removing $F \times(-1,1)$. Let $\mathbf{T}$ and $\mathbf{T}^{\prime}$ be the unions of prime decomposition systems for the components of $N$ as 3-manifolds, and further require that the spheres of $\mathbf{S}$ and $\mathbf{T}$ be disjoint and that the spheres of $\mathbf{S}^{\prime}$ and $\mathbf{T}^{\prime}$ be disjoint.

In order to see that this is possible, consider $\mathbf{S}$ and any prime decomposition system $\mathbf{T}$ for the components of $N$. Assume without loss of generality that the spheres of $\mathbf{S}$ and $\mathbf{T}$ intersect transversely in simple closed curves. Let $D$ be an innermost disk in some sphere $S \in \mathbf{S}$, where there exists some sphere $T \in \mathbf{T}$ such that $\partial D \subset T$, and $\operatorname{Int} D$ does not meet any sphere of $\mathbf{T}$. Let $T_{1}$ and $T_{2}$ be the two 2-spheres obtained by surgering $T$ along $D$ and pushing slightly off of $T$ and $S$. Both $T_{1}$ and $T_{2}$ separate $N$ (and $M$ ) by hypothesis. In the factor $N^{\prime}$ of $N$ which contains $T_{1}$ and $T_{2}$, exactly one of these two 2spheres, say $T_{1}$, must bound a 3-ball which contains the other. If we replace $T$ by $T_{1}$, then we obtain a new prime decomposition system for $N$ which meets $\mathbf{S}$ in fewer simple closed curves. Continue in this fashion until the systems are disjoint.

Since $\mathbf{T}$ and $\mathbf{T}^{\prime}$ are prime decomposition systems for the components of $N$, there is a one-to-one correspondence between the factors $N_{1}, \ldots, N_{n}$ under $\mathbf{T}$ and the factors $N_{1}^{\prime}, \ldots, N_{n}^{\prime}$ under $\mathbf{T}^{\prime}$ such that for every $i$ there exists an (orientation-preserving) homeomorphism $h_{i}: N_{i} \rightarrow N_{i}^{\prime}$ which is the identity on $\partial N_{i}$. For those factors $N_{i}$ and $N_{i}^{\prime}$ whose boundaries meet Int $M$, which occurs where the bicollar of $F$ was removed, reattach those components of the bicollar which meet $N_{i}$ and $N_{i}^{\prime}$, and extend $h_{i}$ as the identity across the bicollar. This may involve the merging of some $N_{i}$ 's into single 3-manifolds. We obtain 3-manifolds $R_{1}, \ldots, R_{m}$ and $R_{1}^{\prime}, \ldots, R_{m}^{\prime}$ which result from cutting $M$ along $\mathbf{T}$ and $\mathbf{T}^{\prime}$ respectively and then capping off with 3-balls, and we also obtain homeomorphisms $g_{i}: R_{i} \rightarrow R_{i}^{\prime}$ which are identities on $\partial R_{i}$ and on $F \cap R_{i}$. For every $i$, let $\mathbf{S}_{i}$ and $\mathbf{S}_{i}^{\prime}$ consist of those spheres of $\mathbf{S}$ and $\mathbf{S}^{\prime}$ which are contained in $R_{i}$ and $R_{i}^{\prime}$ respectively.

Suppose that there is some $R_{i}$ which does not meet $F$. Let $X_{i}$ be the closure of the component of $M-U \mathrm{~T}$ which when capped off with 3-balls yields $R_{i}$. Let $T \in \mathbf{T}$ be one of the spheres in $\partial X_{i}$. Let $\left(M_{*}, F_{*}\right)$ be the factor of $\mathbf{F}(M, F ; \mathbf{S})$ which contains $T$. If $T$ were to bound a 3-ball in $\left(M_{*}, F_{*}\right)$ which did not meet $F_{*}$, then necessarily it would bound a 3-ball in $M$ which did not meet $F$, since the only thing to prevent it from bounding such a 3-ball would be the presence of other spheres of $\mathbf{S}$ on the appropriate side of $T$, which would imply that $F$ meets $M_{*}$ on that side of $T$. Thus $X_{i}$ would be a punctured 3-sphere, and $R_{i}$ would be a 3-sphere, contradicting the nontriviality of $\mathbf{T}$. Therefore $T$ does not bound such a 3-ball in $\left(M_{*}, F_{*}\right)$. By the previous lemma, $\left(M_{*}, F_{*}\right)$ must be one of two possible types, and since $X_{i} \subset M_{*}$ cannot be a punctured 3-sphere, it must be the case that $M_{*}$ is a prime 3-manifold, $F_{*}$ is a 2-sphere, and $T$ bounds a 3-ball in $M_{*}$ containing 
$F_{*}$. All of the above applies equally to $R_{i}^{\prime}=g_{i}\left(R_{i}\right)$, and therefore we may convert $g_{i}$ to a homeomorphism $f_{i}:\left(M_{*}, F_{*}\right) \rightarrow\left(M_{*}^{\prime}, F_{*}^{\prime}\right)$. The same process should be carried out for all such $R_{i}$. Before leaving this case, let us note a few additional facts. First, there is only one sphere of $\mathbf{T}$ in $\partial X$ since all such spheres are in $M_{*}$ and due to the fact that $M_{*}$ is prime, the presence of more than one such sphere would lead to the fact that some component of $N-\cup \mathrm{T}$ is a punctured 3-sphere, contradicting nontriviality of $\mathbf{T}$. Second, let $Y_{j}$ be the closure of the component of $M-\bigcup T-\bigcup S$ on the other side of $T$, contained in some $R_{j}$. Clearly the factor of $\left(R_{j}, F \cap R_{j}\right)$ under $\mathbf{S}_{j}$ which contains $Y_{j}$ must be the trivial factor $\left(S^{3}, S^{2}\right)$ since $T$ bounds a 3-ball in $M_{*}$ containing the 2-sphere $F_{*}$. The same applies of course to $R_{j}^{\prime}$.

Let $\mathbf{T}_{0}$ and $\mathbf{T}_{0}^{\prime}$ consist of those spheres of $\mathbf{T}$ and $\mathbf{T}^{\prime}$ which are not contained in the boundary of components $X_{i}$ and $X_{i}^{\prime}$ of the type described above. For each such sphere of $\mathrm{T}_{0}$, the $R_{i}$ and $R_{j}$ on either side must both meet $F$, and likewise for $\mathbf{T}_{0}^{\prime}$. Since the number of $R_{i}$ 's which meet $F$ is the same as the number of $R_{i}$ 's which meet $F$, the number of spheres in $\mathbf{T}_{0}$ and $\mathbf{T}_{0}^{\prime}$ must be the same. Let $T$ be any sphere of $\mathrm{T}_{0}$, with $R_{i}$ and $R_{j}$ on either side, and let $\left(M_{*}, F_{*}\right)$ be the factor of $\mathbf{F}(M, F ; \mathbf{S})$ which contains $T$. Since $R_{i}$ and $R_{j}$ must both meet $F$, necessarily both components of $M_{*}-T$ must meet $F_{*}$. By the previous lemma, $M_{*}=S^{3}$ and $F_{*}$ consists of two disjoint 2-spheres. Note that no other sphere of $\mathbf{T}$ can be contained in $M_{*}$, since this would induce a punctured 3-sphere component of $N-\cup \mathbf{T}$, contradicting nontriviality of $\mathbf{T}$. Thus there is one such factor of $\mathbf{F}(M, F ; \mathbf{S})$ for each sphere of $\mathbf{T}_{0}$, and one such factor of $\mathbf{F}\left(M, F ; \mathbf{S}^{\prime}\right)$ for each sphere of $\mathbf{T}_{0}^{\prime}$. Since the number of spheres in $\mathbf{T}_{0}$ and $\mathbf{T}_{0}^{\prime}$ is the same, there is a one-to-one correspondence between such factors under $\mathbf{S}$ and $\mathbf{S}^{\prime}$, and corresponding factors are clearly homeomorphic. Now, let $Y_{i}$ be the closure of the component of $M-\bigcup \mathrm{T}-\bigcup \mathbf{S}$ on either side of $T$, contained in some $R_{i}$. Clearly the factor of $\left(R_{i}, F \cap R_{i}\right)$ under $\mathbf{S}_{i}$ which contains $Y_{i}$ must be the trivial factor $\left(S^{3}, S^{2}\right)$ since on this side $T$ bounds a 3-ball in $M_{*}$ containing one of the two 2-spheres of $F_{*}$. This is also true on the other side of $T$, and also for spheres of $\mathbf{T}_{0}^{\prime}$.

Now any factor $\left(M_{*}, F_{*}\right)$ of $\mathbf{F}(M, F ; \mathbf{S})$ which is not one of the ones considered above must contain no spheres of $\mathbf{T}$. Thus it is a factor of some $\mathbf{F}\left(R_{i}, F \cap R_{i} ; \mathbf{S}_{i}\right)$. Consider any such $R_{i}$ and $R_{i}^{\prime}$ with homeomorphism $g_{i}$ : $R_{i} \rightarrow R_{i}^{\prime}$. Let $F_{i}=F \cap R_{i}$ and $F_{i}^{\prime}=F^{\prime} \cap R_{i}^{\prime}$. Recall that $g_{i}: F_{i} \rightarrow F_{i}^{\prime}$ is the identity. $\mathbf{S}_{i}$ provides a decomposition of $\left(R_{i}, F_{i}\right)$ into factors which are prime or trivial, since the existence of any nontrivial decomposition sphere for $\left(R_{i}, F_{i}\right)$ would contradict the fact that $\mathbf{S}$ was a prime decomposition system. Those factors of $\left(R_{i}, F_{i}\right)$ which are trivial are exactly those which arise from splitting factors of $\mathbf{F}(M, F ; \mathbf{S})$ along spheres of $T$. Thus there is a one-to-one correspondence between factors of $\mathbf{F}(M, F ; \mathbf{S})$ which do not meet spheres of $T$ and the prime factors of the various $\mathbf{F}\left(R_{i}, F_{i} ; \mathbf{S}_{i}\right)$. Drop any unnecessary spheres from $\mathbf{S}_{i}$ and $\mathbf{S}_{i}^{\prime}$, one for each trivial factor. By Theorem 4.6 there is a one-to-one correspondence between the factors of $\left(R_{i}^{\prime}, F_{i}^{\prime}\right)$ under each of $\mathbf{S}^{\prime}$ and $g_{i}(\mathbf{S})$, which are not copies of $\left(S^{3}, T^{2}\right)$, such that corresponding factors are stably equivalent. This implies such a correspondence between $\mathbf{F}\left(R_{i}, F_{i} ; \mathbf{S}_{i}\right)$ and $\mathbf{F}\left(R_{i}^{\prime}, F_{i}^{\prime} ; \mathbf{S}_{i}^{\prime}\right)$ for every $i$, and therefore there is such a correspondence between all factors of $(M, F)$ under each of $\mathbf{S}$ and $\mathbf{S}^{\prime}$ which have not previously been dealt with. 

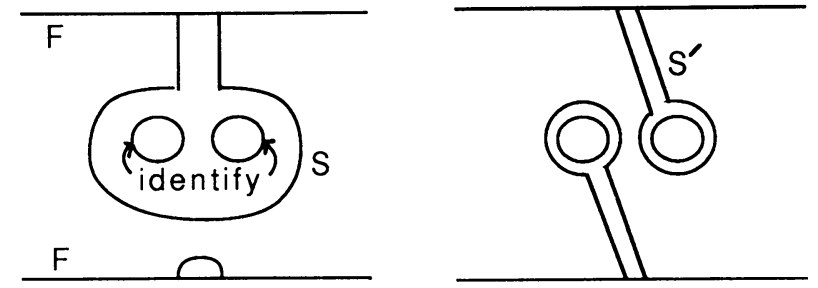

FIGURE 2

Therefore $(M, F)$ has unique prime decompositions up to stable equivalence.

We conclude this section with an example which demonstrates that nonseparating 2-spheres in $M-F$ do cause problems. Let $F$ be a 2-manifold, and identify $F$ with a fiber of $F \times S^{1}$. Let $B$ be a 3-ball in $F \times S^{1}$ disjoint from $F$. Let $M$ be the connected sum of $F \times S^{1}$ and $S^{2} \times S^{1}$, where $B$ is the 3-ball in $F \times S^{1}$ whose interior is removed to form the connected sum. We consider the surface $(M, F)$, noting that $M-F$ contains nonseparating 2-spheres, and construct two different prime decompositions of $(M, F)$ (see Figure 2). For the first prime decomposition, let $S$ be one boundary component of a small regular neighborhood of $\partial B \cup \alpha$, where $\alpha$ is an arc from $\partial B$ to $F$. For the second prime decomposition, let $S_{0}$ be a nonseparating 2-sphere of $M-F$, let $\beta$ be a simple closed curve which meets each of $F$ and $S_{0}$ transversely in a single point, and let $S^{\prime}$ be the boundary of a regular neighborhood of $S_{0} \cup \beta$. In each decomposition, one of the factors is $\left(F \times S^{1}, F\right)$, and the other factor is of the form $\left(S^{2} \times S^{1}, G\right)$ where $G$ is a 2-sphere. In the first decomposition, $G$ bounds a 3 -ball in $S^{2} \times S^{1}$, and in the second decomposition, $G$ is a nonseparating sphere in $S^{2} \times S^{1}$. Since stabilization can never change a separating surface to a nonseparating surface or vice versa, the factors of these prime decompositions are not stably equivalent.

Question. Does Theorem 4.6 remain valid if the nonseparating 2-sphere hypothesis is dropped, or does there exist a counterexample for this case? Note that in this situation, any nonseparating 2-sphere must intersect $F$.

\section{STABle EQUiVAlENCE VERSUS EQUiVAlENCE IN THE 3-SPHERE}

In [Su], Suzuki raised the following question: if two surfaces in $S^{3}$ are prime and stably equivalent, does it follow that they are isotopic? In other words, can his main theorem (stated here as Theorem 1.2) be strengthened to say that the factors in any prime decomposition of a connected surface $\left(S^{3}, F\right)$ are unique up to ambient isotopy? In this section, we will demonstrate that the answer is "no" when the surfaces are of genus 3 or greater. We begin by proving that stable isotopy does imply ambient isotopy for genus less than 3 . Throughout this section, we will write surfaces $\left(S^{3}, F\right)$ as just $F$.

Theorem 5.1. Let $F$ and $F^{\prime}$ be connected surfaces in $S^{3}$ of the same genus $g \leq 2$. If $F$ and $F^{\prime}$ are stably isotopic, then they are ambient isotopic.

Proof. Note that $S^{3}-F$ and $S^{3}-F^{\prime}$ are irreducible. Thus, by Theorem 3.3, we may assume without loss of generality that $F$ and $F^{\prime}$ have the same maximal 
triad $\left(T ; B_{1}, B_{2}\right)$. If $T=S^{3}$, then $F$ and $F^{\prime}$ are both Heegaard splittings of $S^{3}$, and by [W] they must be ambient isotopic. If either $B_{1}$ or $B_{2}$ is connected of genus $g$, say $B_{1}$, then the compression bodies $W$ and $W^{\prime}$ which have interior boundary $B_{1}$ and exterior boundaries $F$ and $F^{\prime}$ respectively must be simply collars of $B_{1}$, and therefore $F$ and $F^{\prime}$ are both ambient isotopic to $B_{1}$ and thus to each other. If either $g=0$ or $g=1$, then one of the above circumstances must occur.

Now suppose that $g=2$, that $T \neq S^{3}$, and that neither of $B_{1}$ or $B_{2}$ is a connected surface of genus 2 . This means that both $F$ and $F^{\prime}$ must have compressing disks on each side. By [T], a genus 2 surface in $S^{3}$ which compresses on both sides is not prime, and therefore $F$ and $F^{\prime}$ have nontrivial decomposition spheres $S$ and $S^{\prime}$ respectively which are contained in $T$. Since the factors of $T$ under $S$ or $S^{\prime}$ have Heegaard splittings of genus 1, they must be prime, and thus it is possible to isotope $S^{\prime}$ to $S$ within $T$. So we will assume that $S=S^{\prime}$. Let $\left(T_{i} ; C_{i 1}, C_{i 2}\right)$ for $i=1,2$ be the factors of $T$ under the decomposition given by $S$. Also let $G_{1}$ and $G_{2}$ be the factors of $F$ given by $S$, and $G_{1}^{\prime}$ and $G_{2}^{\prime}$ be the factors of $F^{\prime}$, all of these necessarily of genus 1 . Since each $T_{i}$ is a maximal triad for both $G_{i}$ and $G_{i}^{\prime}$, and these surfaces must compress on at least one side, $T_{i}$ is either a solid torus or $S^{3}$. For the same reasons as cited above, $G_{1}$ must be ambient isotopic to $G_{1}^{\prime}$ within $T_{1}$ and $G_{2}$ must be ambient isotopic to $G_{2}^{\prime}$ within $T_{2}$. Therefore $F$ is ambient isotopic to $F^{\prime}$ in $T$ and thus in $S^{3}$.

In order to obtain examples of inequivalent surfaces in $S^{3}$, of genus 3 or higher, which are stably isotopic, consider the work of [Mo2 and BRZ]. Let $V$ be a solid torus in $S^{3}$ which is a regular neighborhood of a $(p, q)$ torus knot with $2 \leq p<q$, and let $X$ be the closure of the complement of $V$. These papers demonstrate that $(X ; \varnothing, \partial X)$ has at least two inequivalent Heegaard splittings of genus 2 if $p+1<q$, and has three inequivalent Heegaard splittings of genus 2 if $q \not \equiv \pm 1(\bmod p)$. Let $H \subset V$ be a handlebody of genus $g \geq 1$, which meets $\partial V$ in a disk, and such that the closure of $V-H$ has incompressible boundary. Let $M=X \cup H ; M$ is a submanifold of $S^{3}$. We can manufacture Heegaard splittings of $M$ in the following way: let $G$ be a surface in Int $H$ parallel to $\partial H$, let $F_{0}$ be a genus 2 Heegaard splitting of $X$, and form a surface $F$ by connecting $G$ to $F_{0}$ by a "nice" tube in $M$, where by "nice" we mean an annulus that is vertical in the collar of $\partial X$ in an optimal 1-handle decomposition of the compression body with interior boundary $\partial X$ and exterior boundary $F_{0}$. Then $F$ is a Heegaard splitting of $M$ of genus $2+g \geq 3$, and in fact $(M ; \varnothing, \partial M)$ is the maximal triad of $F$ due to the fact that the closure of $S^{3}-M=V-H$ has incompressible boundary. If we start with an inequivalent Heegaard splitting $F_{0}^{\prime}$ of $X$, then we obtain a Heegaard splitting $F^{\prime}$ of $M$ which is also of genus $2+g$. Since $F$ and $F^{\prime}$ are both Heegaard splittings of $M$, they are stably isotopic in $M$, and thus in $S^{3}$. Next, it is possible to show that $F$ and $F^{\prime}$ are inequivalent in $M$ by the same techniques used by Moriah in [Mol] to show that $F_{0}$ and $F_{0}^{\prime}$ are inequivalent in $X$ (it does not appear possible to directly apply the techniques used to prove inequivalence in [Mo2 or BRZ]). Finally, inequivalence of $F$ and $F^{\prime}$ in $M$ implies inequivalence in $S^{3}$ since $M$ is the unique maximal triad for both $F$ and $F^{\prime}$. Therefore $F$ and $F^{\prime}$ are inequivalent surfaces in $S^{3}$ of the same genus (greater than 2) which are stably isotopic. 
Suppose that such a Heegaard splitting $F$ was not a prime surface in $S^{3}$. Then there would exist a nontrivial decomposition sphere $S$ for $F$ contained in $M$, since $M$ is the maximal triad of $F . S$ bounds a 3-ball in $M$ due to the fact that $M$ is irreducible, and therefore $F$ is a stabilization of some Heegaard splitting $F^{*}$ of $M$ which is of genus $1+g$, the same genus as $\partial M$. This is impossible since it would require $F^{*}$ to be parallel to $\partial M$, and $M$ is not a handlebody. Therefore $F$ must be prime.

If $F^{\prime \prime}$ is a stabilization of $F$ and $F^{\prime}$, then $F^{\prime \prime}$ has prime decompositions whose factors on the one hand consist of $F$ and copies of $\left(S^{3}, T^{2}\right)$, and on the other hand consist of $F^{\prime}$ and copies of $\left(S^{3}, T^{2}\right)$. But $F$ and $F^{\prime}$ are only stably isotopic, not ambient isotopic. Therefore the answer to Suzuki's question is "no."

\section{REFERENCES}

[Bo] F. Bonahon, Cobordism of automorphisms of surfaces, Ann. Sci. Ecole Norm. Sup. 1 (1983), 237-270.

[BoO] F. Bonahon and J.-P. Otal, Scindements de Heegaard des espaces lenticulaires, Ann. Sci. Ecole Norm. Sup. 16 (1983), 451-466.

[BRZ] M. Boileau, M. Rost, and H. Zieschang, On Heegaard decompositions of torus knot exteriors and related Seifert fibered spaces, Math. Ann. 279 (1988), 553-581.

[CG] A. J. Casson and C. McA. Gordon, Reducing Heegaard splittings, Topology Appl. 27 (1987), 275-283.

[Cr] R. Craggs, A new proof of the Reidemeister-Singer theorem on stable equivalence of Heegaard splittings, Proc. Amer. Math. Soc. 57 (1976), 143-147.

[Ha] W. Haken, Some results on surfaces in 3-manifolds, Studies in Modern Topology, Math. Assoc. Amer., Prentice-Hall, Englewood Cliffs, N. J., 1968, pp. 34-98.

[He] J. Hempel, 3-manifolds, Ann. of Math. Studies, no. 86, Princeton Univ. Press, Princeton, N. J., 1976.

[Ja] W. Jaco, Lectures on three-manifold topology, CBMS Regional Conf. Ser. in Math., no. 43, Amer. Math. Soc., Providence, R. I., 1980.

[Mo1] Y. Moriah, Heegaard splittings and group presentations, Dissertation, Univ. of Texas at Austin, 1986.

[Mo2] _ Heegaard splittings of Seifert fibered spaces, Invent. Math. 91 (1988), 465-481.

[M] M. Motto, Surfaces in three-manifolds and three-manifold triads, Dissertation, Univ. of Texas at Austin, 1988.

[Re] K. Reidemeister, Zur dreidimensionalen Topologie, Abh. Math. Sem. Univ. Hamburg 9 (1933), 189-194.

[Sie] L. C. Siebenmann, Les bisections expliquent le théorème de Reidemeister-Singer, Publications Université de Paris-Sud, Département de Mathématique, 1979.

[Sin] J. Singer, Three dimensional manifolds and their Heegaard diagrams, Trans. Amer. Math. Soc. 35 (1933), 88-111.

[Su] S. Suzuki, On surfaces in 3-sphere: stable congruence, Math. Japon. 20 (1975), 65-83.

[T] Y. Tsukui, On a prime surface of genus 2 and homeomorphic splitting of 3-sphere, Yokohama Math. J. 23 (1975), 63-75.

[W] F. Waldhausen, Heegaard-Zerlegungen der 3-Sphäre, Topology 7 (1968), 195-203.

Department of Mathematics, Indiana University, Bloomington, Indiana 47405

Current address: Department of Mathematical Sciences, Ball State University, Muncie, Indiana 47306

E-mail address:00mhmotto@bsuvax1.bitnet 